Göteborg

ITP $92-56$

December 1992

\title{
An Algorithm for Computing Four-Ramond Vertices at Arbitrary Level
}

\author{
Niclas Engberg巾 \\ Bengt E.W Nilsson? \\ Per Sundellp \\ Institute of Theoretical Physics \\ Chalmers University of Technology \\ and University of Göteborg \\ S-412 96 Göteborg, Sweden
}

\begin{abstract}
We perform the sewing of two (dual) Ramond reggeon vertices and derive an algorithm by means of which the so obtained four-Ramond reggeon vertex may be explicitly computed at arbitrary oscillator (mass) level. A closed form of the four-vertex is then deduced on the basis of a comparison to all terms obtained by sewing that contain only level zero and one oscillators. Results are presented for both complex fermions and for the previously studied case of real fermions.
\end{abstract}

\footnotetext{
${ }^{1}$ TFENE@FY.CHALMERS.SE

${ }^{2}$ TFEBN@FY.CHALMERS.SE

${ }^{3}$ TFEPSU@FY.CHALMERS.SE
} 


\section{Introduction}

Out of the large body of explicitly known expressions for bosonic and fermionic string scattering amplitudes only a small subset is understood from the point of view of operator sewing. Using reggeonic operator vertices it is relatively straightforward to derive such amplitudes for any genus, $g$, and number of external legs, $N$, provided only fields with periodic (untwisted) boundary conditions in the complex plane are considered [1-7]. As soon as one incorporates twisted fields, either bosons or fermions, e.g. the Ramond sector of the NSR string, one encounters severe technical problems. These problems are obvious already in the early literature on this subject [8-18] (see also [19-21]), and have not yet been overcome. Although there exist in many specific cases means of getting around these obstacles, e.g. BPZ techniques [22, bosonization [23], overlap conditions [24, 25], path integrals [26, 27], other operator formalisms [28] etc, it would still be of great value to find a way to derive string vertices as well as conformal field theory (CFT) correlation functions directly by sewing of some basic three-vertices [29-32,3], or, which is equivalent but sometimes more convenient, dual vertices [33-35,7].

In the case of the NSR string the problems eluded to above set in already when trying to compute one of the most fundamental vertices namely the four-Ramond reggeon vertex, i.e. the vertex describing the scattering of four fermions from the spacetime target space point of view. This vertex, usually discussed in terms of real world sheet fermions, has been the main object of interest in a large number of previous works (see e.g. [8, 10, 11, 12, 14, 17]) and will only be commented upon towards the end of this paper. Instead we concentrate here on the slightly simpler case based on complex fermions for which the results presented are quite detailed. The reason for doing this is that the main technical problems to be addressed here are basically independent of whether we use real or complex fields, and that most of our results are somewhat easier to derive and to present in terms of complex fields. It deserves to be emphasized, however, that the same kind of results are easily extracted also for the standard NSR case based on real fermions and we collect the formulae for this case in section 5 .

Another reason for being interested in the case of complex fermions is the possibility of normal ordering also the zero modes of the twisted fields which is not possible in the real case. 
In fact, the starting point for our investigations is an expression for the dual Ramond reggeon vertex which is independent of the normal ordering chosen and of whether we use complex or real fermion fields. The sewing computations as well as the complications that arise when trying to put the results into closed form are essentially the same as in the case of the standard four-Ramond vertex, and our main goal is to extend significantly on previous sewing results in a simpler setting and to propose closed answers for both complex and real four-reggeon vertices. The form of these closed answers are inspired by three-vertices previously discussed by LeClair in [36]. A generalization of these latter vertices to an arbitrary number of $N S$ and $R$ legs was subsequently suggested in [37], but was only compared to the sewed operator expression for the one non-zero term containing only zero modes. However, with our new results we can compute the four-vertex at any oscillator (mass) level and are thus in effect able to prove that the proposed answer is the only possible one. (A detailed comparison is carried out below for all terms containing only level one and/or zero oscillators.) A step by step derivation of the proposed closed form of the four-vertex is a more difficult task that we hope to come back to in a future publication. We also hope to be able to generalize this use of complex fields to other CFT's and thereby extend this formalism to cases where sewing has not yet been attempted.

The paper is organized as follows. In section 2 we set up the formalism and present a form of the dual twisted (Ramond) reggeon vertex from which one can deduce both the real and the complex vertices of interest to this paper. The explicit form of these vertices are given at the end of that section. The complex one is then used, in section 3, to sew together two dual twisted reggeon vertices. The so obtained four-reggeon vertex is expressed in terms of an infinite set of quantities constructed from infinite dimensional matrices and vectors. These quantities are then studied and an algorithm presented by means of which they may all be computed. Explicit answers are derived for a large number of them which are then used, in section 4, to deduce the closed form of the twisted four-reggeon vertex. Formulae extending our conclusions to three other closely related vertices for complex fermions as well as to the standard four-Ramond reggeon vertex (derived using real fermions) together with some other relevant facts are collected in section 5. Some further comments and conclusions are given in section 6 . 


\section{Background and notation}

All previous sewing results in connection with reggeon four-vertices for external twisted fermionic fields have been obtained using the real fermions ( "^" denotes operator valued quantities)

$$
\begin{aligned}
\hat{\psi}_{N S}^{\mu}(z) & =i \sum_{r \in \mathbf{Z}+\frac{1}{2}} \hat{\psi}_{r}^{\mu} z^{-r-\frac{1}{2}} \quad, \quad \mu=1, \ldots, D \\
\hat{\psi}_{R}^{\mu}(z) & =i \sum_{m \in \mathbf{Z}} \hat{\psi}_{m}^{\mu} z^{-m-\frac{1}{2}}
\end{aligned}
$$

whose Laurent modes satisfy the hermiticity conditions

$$
\left(\hat{\psi}_{r}^{\mu}\right)^{\dagger}=\hat{\psi}_{-r}^{\mu}, \quad\left(\hat{\psi}_{m}^{\mu}\right)^{\dagger}=\hat{\psi}_{-m}^{\mu}
$$

Our notation is such that indices $r, s$ always take values in the set $\mathbf{Z}+\frac{1}{2}$, while $m, n$ always refer to integers. The vacua in the two sectors, usually referred to as Neveu-Schwarz $(N S)$ and Ramond $(R)$, respectively, are defined by

$$
\hat{\psi}_{r}^{\mu}|0\rangle=0, \quad \hat{\psi}_{n}^{\mu}|\alpha\rangle=0 ; \quad r \geq \frac{1}{2}, \quad n \geq 1
$$

where $|\alpha\rangle$ is a set of degenerate states carrying a representation of the Clifford algebra satisfied by the Ramond zero modes $\hat{\psi}_{0}^{\mu}$. To define the systems of main interest here we assume $D$ to be an even integer, say $D=2 d$, and combine pairs of the real fields into complex fields as follows

$$
\begin{aligned}
\hat{\psi}_{N S}^{a}(z) & =\frac{1}{\sqrt{2}}\left(\hat{\psi}_{N S}^{a}(z)+i \hat{\psi}_{N S}^{a+d}(z)\right), \quad \hat{\bar{\psi}}_{N S}^{a}(z)=\frac{1}{\sqrt{2}}\left(\hat{\psi}_{N S}^{a}(z)-i \hat{\psi}_{N S}^{a+d}(z)\right), \\
\hat{\psi}_{R}^{a}(z) & =\frac{1}{\sqrt{2}}\left(\hat{\psi}_{R}^{a}(z)+i \hat{\psi}_{R}^{a+d}(z)\right), \quad \hat{\bar{\psi}}_{R}^{a}(z)=\frac{1}{\sqrt{2}}\left(\hat{\psi}_{R}^{a}(z)-i \hat{\psi}_{R}^{a+d}(z)\right),
\end{aligned}
$$

where the index $a$ runs over values $1, \ldots, d$. These complex fields are expanded as

$$
\begin{aligned}
\hat{\psi}_{N S}^{a}(z) & =i \sum_{r \in \mathbf{Z}+\frac{1}{2}} \hat{\psi}_{r}^{a} z^{-r-\frac{1}{2}}, \quad \hat{\bar{\psi}}_{N S}^{a}(z)=i \sum_{r \in \mathbf{Z}+\frac{1}{2}} \hat{\bar{\psi}}_{r}^{a} z^{-r-\frac{1}{2}} \\
\hat{\psi}_{R}^{a}(z) & =i \sum_{m \in \mathbf{Z}} \hat{\psi}_{m}^{a} z^{-m-\frac{1}{2}}, \quad \hat{\bar{\psi}}_{R}^{a}(z)=i \sum_{n \in \mathbf{Z}} \hat{\bar{\psi}}_{n}^{a} z^{-n-\frac{1}{2}}
\end{aligned}
$$

where the respective modes satisfy the hermiticity properties

$$
\left(\hat{\psi}_{r}^{a}\right)^{\dagger}=\hat{\bar{\psi}}_{-r}^{a}, \quad\left(\hat{\bar{\psi}}_{r}^{a}\right)^{\dagger}=\hat{\psi}_{-r}^{a}, \quad\left(\hat{\psi}_{m}^{a}\right)^{\dagger}=\hat{\bar{\psi}}_{-m}^{a}, \quad\left(\hat{\bar{\psi}}_{m}^{a}\right)^{\dagger}=\hat{\psi}_{-m}^{a}
$$


It is now convenient to summarize all non-zero oscillator commutation relations as follows:

$$
\left\{\hat{\psi}_{N S}^{a(+)}(z), \hat{\bar{\psi}}_{N S}^{b(-)}(w)\right\}=\left\{\hat{\bar{\psi}}_{N S}^{a(+)}(z), \hat{\psi}_{N S}^{b(-)}(w)\right\}=-\frac{\delta^{a b}}{z-w}, \quad|z|>|w|
$$

in the $N S$ sector,

$$
\left\{\hat{\psi}_{R}^{a(+)}(z), \hat{\bar{\psi}}_{R}^{b(-)}(w)\right\}=-\sqrt{\frac{z}{w}} \frac{\delta^{a b}}{z-w}, \quad\left\{\hat{\bar{\psi}}_{R}^{a(+)}(z), \hat{\psi}_{R}^{b(-)}(w)\right\}=-\sqrt{\frac{w}{z}} \frac{\delta^{a b}}{z-w},|z|>|w|
$$

in the $R$ sector. Here we have defined creation () and annihilation (+) fields by the following expansions:

$$
\begin{gathered}
\hat{\psi}_{N S}^{a(+)}(z)=i \sum_{r=\frac{1}{2}}^{\infty} \hat{\psi}_{r}^{a} z^{-r-\frac{1}{2}}, \quad \hat{\psi}_{N S}^{a(-)}(z)=i \sum_{r=\frac{1}{2}}^{\infty} \hat{\psi}_{-r}^{a} z^{r-\frac{1}{2}}, \\
\hat{\bar{\psi}}_{N S}^{a(+)}(z)=i \sum_{r=\frac{1}{2}}^{\infty} \hat{\bar{\psi}}_{r}^{a} z^{-r-\frac{1}{2}}, \quad \hat{\bar{\psi}}_{N S}^{a(-)}(z)=i \sum_{r=\frac{1}{2}}^{\infty} \hat{\bar{\psi}}_{-r}^{a} z^{r-\frac{1}{2}}, \\
\hat{\psi}_{R}^{a(+)}(z)=i \sum_{n=0}^{\infty} \hat{\psi}_{n}^{a} z^{-n-\frac{1}{2}}, \quad \hat{\psi}_{R}^{a(-)}(z)=i \sum_{n=1}^{\infty} \hat{\psi}_{-n}^{a} z^{n-\frac{1}{2}}, \\
\hat{\bar{\psi}}_{R}^{a(+)}(z)=i \sum_{n=1}^{\infty} \hat{\bar{\psi}}_{n}^{a} z^{-n-\frac{1}{2}}, \quad \hat{\bar{\psi}}_{R}^{a(-)}(z)=i \sum_{n=0}^{\infty} \hat{\bar{\psi}}_{-n}^{a} z^{n-\frac{1}{2}}
\end{gathered}
$$

which explains the different square root factors appearing on the right hand sides of the $R$ commutators in eq. (10) as due to the $R$ zero mode. The ket vacua of the complex fermions can thus be defined through the equations

$$
\begin{aligned}
& \hat{\psi}_{N S}^{a(+)}(z)|0\rangle=\hat{\bar{\psi}}_{N S}^{a(+)}(z)|0\rangle=0 \\
& \hat{\psi}_{R}^{a(+)}(z)|0\rangle=\hat{\bar{\psi}}_{R}^{a(+)}(z)|0\rangle=0
\end{aligned}
$$

while for the bra vacua the corresponding equations follow from hermitian conjugation of the oscillators and $\langle 0|=(|0\rangle)^{\dagger}$. Note that notationally we do not distinguish between the NS vacuum and the Clifford representation vacuum in the complex $R$ sector.

By introducing two $N S$ type normal ordering fields $\hat{\psi}_{1}$ and $\hat{\psi}_{2}$ together with one $N S$ type auxiliary field $\hat{\psi}_{\text {aux }}$ the basic (dual) vertex can be written, in either the real or complex $N S$ and $R$ sectors, in the following forms:

$$
\begin{aligned}
\hat{W}_{\text {ext }}(V) & ={ }_{1}\left\langle\left. 0\right|_{2}\left\langle 0\left|e^{\oint_{C} d z\left(\hat{\psi}_{a u x}^{\mu}+i \hat{\psi}_{1}^{\mu}\right)(z)\left(\hat{\psi}_{e x t}^{\mu}+i \hat{\psi}_{2}^{\mu}\right)^{-1}(z)}\right| 0\right\rangle_{2} \mid 0\right\rangle_{1}= \\
& ={ }_{1}\left\langle 0\left|{ }_{2}\langle 0| e^{\oint_{C} d z\left(\left(\hat{\psi}_{a u x}^{a}+i \hat{\psi}_{1}^{a}\right)(z)\left(\hat{\bar{\psi}}_{e x t}^{a}+i \hat{\bar{\psi}}_{2}^{a}\right)^{V^{-1}}(z)+\left(\hat{\bar{\psi}}_{a u x}^{a}+i \hat{\bar{\psi}}_{1}^{a}\right)(z)\left(\hat{\psi}_{\text {ext }}^{a}+i \hat{\psi}_{2}^{a}\right)^{V^{-1}}(z)\right.}\right) \mid 0\right\rangle_{2}|0\rangle_{1}(
\end{aligned}
$$


where $V$ denotes a projective transformation and where the contour $C$ encircles the poles of the $N S$ fields indexed aux and 1, and where the index ext stands for either $N S$ or $R$. Furthermore, the explicit normal ordering of the auxiliary and external fields is induced by performing the correlation functions for the two normal ordering fields as indicated in the equation for the vertex. Note that the complex form (16) is only defined when $D$ is even, and in that case (15) equals (16).

It is convenient to present the vertex in this form for several reasons. One point is connected to the transformation properties of the vertex. When computing e.g. vertices at tree level one has to ensure that the emission points of the external legs are distinct. Multilegged vertices are derived by computing a correlation in the auxiliary fields involving dual vertices; one example is the calculation performed in section three. Now, due to the fact that the normal ordering fields are always (i.e. irrespective of the choice of external legs) of $N S$ type, the necessary transportation of the vertices to different emission points in the complex plane can be accomplished rather easily. By acting with $\hat{\gamma}_{a u x}(V)$, the operator generating the projective transformation $V$, from the left and its inverse from the right, and observing that any projective transformation can be extracted from the $N S$ vacua of the normal ordering field $\hat{\psi}_{1}$, the above form of the vertex is an immediate consequence of the transformation law for the $N S$ fields themselves

$$
\hat{\gamma}_{N S}(V) \hat{\psi}_{N S}(z) \hat{\gamma}_{N S}^{-1}(V)=\sqrt{V^{\prime}(z)} \hat{\psi}_{N S}(V(z))=\hat{\psi}_{N S}^{V}(z)
$$

This equation also defines what we mean by $\hat{\psi}_{N S}^{V}$. As explained in [7] the extra fields must all be of $N S$ type to ensure that the vertex behaves correctly under these transformations.

A second reason for introducing the normal ordering fields is that it clarifies how the different explicit normal ordering prescriptions that are commonly used for the $R$ zero modes are related to each other. In general different options arise because when splitting the fields into two or three parts (e.g. creation, zero modes, and annihilation parts) this can be done in any way one chooses as long as, when the Baker-Hausdorff (BH) formula is used to put the creation and annihilation fields into separate exponentials, one finds that in the commutator term the residue at $z=w$ from the propagator of the aux (ext) field cancels against the corresponding residue from the propagator of the normal ordering field $\hat{\psi}_{1}\left(\hat{\psi}_{2}\right)$. Note that one may leave 
a finite number of modes unordered and still have a well defined vertex. In the NSR string constructed from real fermions this is normally done for the zero modes of the $R$ fields while all other modes are normal ordered the usual way. We denote this particular normal ordering by $\times \underset{\times}{\times}$. In the case of complex fermions one usually normal orders all the modes of the $R$ fields and for this normal ordering we will use the notation : :. Both real and complex NS fields are normal ordered in all their modes as implied here by both the above normal ordering symbols.

For the dual $N S$ vertex both the aux field and the ext field are of $N S$ type. Hence, when computing the correlations functions appearing in the above forms of the dual vertex, using the split in (11) (for complex fermions and the corresponding split for real fermions), one finds that the BH commutator term cancels completely. Doing the same for for vertices with an external twisted field split as in (12) (for complex fermions and the corresponding split for real fermions) one is however left with a remaining term. This term is the actual origin of all the complications we have to face in these twisted cases. To see this explicitly we use the $R$ and $N S$ commutators given in (10) and (9) to compute, in the Ramond case, the total propagator in the $\mathrm{BH}$ commutator term. One finds that it does not vanish but is instead proportional to

$$
\frac{-\sqrt{\frac{w}{z}}+1}{z-w} \text { or } \frac{-\sqrt{\frac{z}{w}}+1}{z-w} \text {, }
$$

which indeed have zero residues. Thus by expressing the projective transformation $V(z)$ in terms of the emission points $z_{1}, z_{2}$ of the external legs (and a parameter $a$ ) through its inverse $V^{-1}(z)=a \frac{z-z_{1}}{z-z_{2}}$, and eliminating the normal ordering fields, the dual reggeon vertex becomes

$$
\begin{aligned}
\hat{W}_{R}(V)= & : e^{\oint_{C} d z\left(\hat{\psi}_{a u x}^{a}(z) \hat{\bar{\psi}}_{R}^{a V^{-1}}(z)+\hat{\bar{\psi}}_{\text {aux }}^{a}(z) \hat{\psi}_{R}^{a V^{-1}}(z)\right)} \\
& e^{-\frac{1}{2} \oint_{C} d z \oint_{C} d w\left(\hat{\psi}_{a u x}^{a}(z) M\left(z_{1}, z_{2} ; z, w\right) \hat{\bar{\psi}}_{a u x}^{a}(w)+\hat{\bar{\psi}}_{a u x}^{a}(z) \bar{M}\left(z_{1}, z_{2} ; z, w\right) \hat{\psi}_{a u x}^{a}(w)\right)}:= \\
= & \times e^{\oint_{C} d z \hat{\psi}_{\text {aux }}^{\mu}(z) \hat{\psi}_{R}^{\mu V^{-1}}(z)} \\
& e^{-\frac{1}{2} \oint_{C} d z \oint_{C} d w \hat{\psi}_{a u x}^{\mu}(z) H\left(z_{1}, z_{2} ; z, w\right) \hat{\psi}_{a u x}^{\mu}(z)} \underset{\times}{\times}
\end{aligned}
$$

when ext equals $R$, and

$$
\begin{aligned}
\hat{W}_{N S}(V)= & =: e^{\oint_{C} d z\left(\hat{\psi}_{a u x}^{a} \hat{\bar{\psi}}_{N S}^{a V^{-1}}(z)+\hat{\bar{\psi}}_{a u x}^{a}(z) \hat{\psi}_{N S}^{a V^{-1}}(z)\right)}:= \\
& =: e^{\oint_{C} d z \hat{\psi}_{a u x}^{\mu}(z) \hat{\psi}_{N S}^{\mu V^{-1}}(z)}:
\end{aligned}
$$


when ext equals $N S$. Here the contours $C$ always encircle the poles of the $\psi_{\text {aux }}$ fields, and : : and $\underset{\times}{\times} \times \underset{\times}{\times}$ refer to the normal ordering of both aux and ext fields. In the four-vertices (19) and (20) we have also defined

$$
\begin{aligned}
M\left(z_{1}, z_{2} ; z, w\right) & =\frac{-\sqrt{\frac{w-z_{1}}{w-z_{2}} \frac{z-z_{2}}{z-z_{1}}}+1}{z-w} \\
\bar{M}\left(z_{1}, z_{2} ; z, w\right) & =\frac{-\sqrt{\frac{z-z_{1}}{z-z_{2}} \frac{w-z_{2}}{w-z_{1}}}+1}{z-w} \\
H\left(z_{1}, z_{2} ; z, w\right) & =\frac{1}{2}\left(M\left(z_{1}, z_{2} ; z, w\right)+\bar{M}\left(z_{1}, z_{2} ; z, w\right)\right)
\end{aligned}
$$

Note that the parameter $a$ cancels in $M$ and $\bar{M}$ and therefore appears in the four-vertices (19) and (20) only in the transported $R$ fields. Let us once again remind the reader that different normal orders are taken into account in (19) and (20) and therefore these to forms look different while in fact being equal (for even $D$ ) as they are both given by the same correlation functions (15) and (16) above.

In the next section we will use the following matrix form of the dual reggeon vertices in the $R$ sector:

$$
\begin{aligned}
& \hat{W}_{R}(V)=: e^{-\hat{\bar{\psi}}_{-r}^{a} U_{r m}^{(-)}(V) \hat{\psi}_{m}^{R, a}-\hat{\bar{\psi}}_{r}^{a} U_{r m}^{(+)}(V) \hat{\psi}_{m}^{R, a}-\hat{\psi}_{-r}^{a} \bar{U}_{r m}^{(-)}(V) \hat{\bar{\psi}}_{m}^{R, a}-\hat{\psi}_{r}^{a} \bar{U}_{r m}^{(+)}(V) \hat{\bar{\psi}}_{m}^{R, a}} \\
& e^{\frac{1}{2}\left(\hat{\bar{\psi}}_{-r}^{a} M_{r s}^{(--)}(V) \hat{\psi}_{-s}^{a}+\hat{\bar{\psi}}_{-r}^{a} M_{r s}^{(-+)}(V) \hat{\psi}_{s}^{a}+\hat{\bar{\psi}}_{r}^{a} M_{r s}^{(+-)}(V) \hat{\psi}_{-s}^{a}+\hat{\bar{\psi}}_{r}^{a} M_{r s}^{(++)}(V) \hat{\psi}_{s}^{a}\right)} \\
& e^{\frac{1}{2}\left(\hat{\psi}_{-r}^{a} \bar{M}_{r s}^{(--)}(V) \hat{\bar{\psi}}_{-s}^{a}+\hat{\psi}_{-r}^{a} \bar{M}_{r s}^{(-+)}(V) \hat{\bar{\psi}}_{s}^{a}+\hat{\psi}_{r}^{a} \bar{M}_{r s}^{(+-)}(V) \hat{\bar{\psi}}_{-s}^{a}+\hat{\psi}_{r}^{a} \bar{M}_{r s}^{(++)}(V) \hat{\bar{\psi}}_{s}^{a}\right)}:= \\
& =\stackrel{\times}{\times} e^{-\hat{\psi}_{-r}^{\mu} U_{r m}^{(-)}(V) \hat{\psi}_{m}^{R, \mu}-\hat{\psi}_{r}^{\mu} U_{r m}^{(+)}(V) \hat{\psi}_{m}^{R, \mu}} \\
& e^{\frac{1}{2}\left(\hat{\psi}_{-r}^{\mu} H_{r s}^{(--)}(V) \hat{\psi}_{-s}^{\mu}+\hat{\psi}_{-r}^{\mu} H_{r s}^{(-+)}(V) \hat{\psi}_{s}^{\mu}+\hat{\psi}_{r}^{\mu} H_{r s}^{(+-)}(V) \hat{\psi}_{-s}^{\mu}+\hat{\psi}_{r}^{\mu} H_{r s}^{(++)}(V) \hat{\psi}_{s}^{\mu}\right) \times} \times
\end{aligned}
$$

where $\hat{\psi}_{r}$ and $\hat{\psi}_{m}^{R}$ are the auxiliary and Ramond Laurent modes respectively, and where we have defined the following matrices

$$
\begin{aligned}
M_{r s}^{( \pm \pm)}(V) & =\oint_{C} d z \oint_{C} d w z^{\mp r-\frac{1}{2}} M\left(z_{1}, z_{2} ; z, w\right) w^{\mp s-\frac{1}{2}} \\
\bar{M}_{r s}^{( \pm \pm)}(V) & =\oint_{C} d z \oint_{C} d w z^{\mp r-\frac{1}{2}} \bar{M}\left(z_{1}, z_{2} ; z, w\right) w^{\mp s-\frac{1}{2}} \\
H_{r s}^{( \pm \pm)}(V) & =\frac{1}{2}\left(M_{r s}^{( \pm \pm)}(V)+\bar{M}_{r s}^{( \pm \pm)}(V)\right) \\
U_{r m}^{( \pm)}(V) & =\bar{U}_{r m}^{ \pm}(V)=\oint_{C} d z z^{\mp r-\frac{1}{2}} \sqrt{V^{-1^{\prime}}(z)}\left(V^{-1}(z)\right)^{-m-\frac{1}{2}}
\end{aligned}
$$


with $C$ encircling zero and infinity. Note that $m, n$ are summed over all integers, while $r, s$ are summed over $\frac{1}{2}, \frac{3}{2}, \ldots$.

In the next section we give the corresponding expression for the sewed complex four-Ramond reggeon vertex and proceed to extract the quantities that we need to compute in order to deduce the closed form (see section 4) of the vertex. In section 5 we will give the equivalent expressions for real Ramond fermions.

\section{The sewed complex four-Ramond reggeon vertex}

The reggeon vertex for four external twisted fermionic (Ramond) complex fields is obtained by computing the auxiliary correlation function

$$
\hat{W}_{R_{1} R_{2}}\left(V_{1}, V_{2}\right)={ }_{\text {aux }}\left\langle 0\left|\hat{W}_{R_{1}}\left(V_{1}\right) \hat{W}_{R_{2}}\left(V_{2}\right)\right| 0\right\rangle_{\text {aux }}
$$

where the dual reggeon vertices are given in (19) (note that each index $R$ refers to two external legs).

Before we proceed we need to specify the projective transformations used in each one of the two vertices. Let us choose the emission points $V(\infty)$ and $V(0)$ to be $\infty$ and $\frac{-1}{\lambda}$, respectively, for vertex 1 , and $-\lambda$ and 0 , respectively, for vertex 2 with $|\lambda|<1$. This can be implemented by the following choice of projective transformations

$$
V_{1}(z)=z-\frac{1}{\lambda}, \quad V_{2}(z)=-\frac{z}{\frac{z}{\lambda}-1}
$$

whose inverses are

$$
V_{1}^{-1}(z)=z+\frac{1}{\lambda}, \quad V_{2}^{-1}(z)=\frac{z}{\frac{z}{\lambda}+1}
$$

This choice implies that the matrix form of the four-vertex becomes (we suppress the spacetime indices henceforth)

$$
\begin{aligned}
& \hat{W}_{R_{1} R_{2}}(\lambda)=:{ }_{a u x}\langle 0| e^{-\hat{\bar{\psi}}_{r} U_{R_{1}, r}^{(+)}-\hat{\psi}_{r} \bar{U}_{R_{1}, r}^{(+)}} e^{\frac{1}{2} \hat{\psi}_{r} M_{r s}^{(++)}\left(V_{1}\right) \hat{\bar{\psi}}_{s}+\frac{1}{2} \hat{\bar{\psi}}_{r} \bar{M}_{r s}^{(++)}\left(V_{1}\right) \hat{\psi}_{s}} \\
& e^{-\hat{\hat{\psi}}_{-r} U_{R_{2}, r}^{(-)}-\hat{\psi}_{-r} \bar{U}_{R_{2}, r}^{(-)}} e^{\frac{1}{2} \hat{\psi}_{-r} M_{r s}^{(--)}\left(V_{2}\right) \hat{\psi}_{-s}+\frac{1}{2} \hat{\psi}_{-r} \bar{M}_{r s}^{(-)}\left(V_{2}\right) \hat{\psi}_{-s}}|0\rangle_{\text {aux }}:
\end{aligned}
$$


where the normal ordering now refers only to the $R$ oscillators in the $U$ 's (see below) and where $\hat{\psi}_{r}$ are the auxiliary modes. Also,

$$
\begin{array}{ll}
M^{(++)}\left(V_{1}\right)=M, & \bar{M}^{(++)}\left(V_{1}\right)=-M^{T} \\
M^{(--)}\left(V_{2}\right)=M^{T}, & \bar{M}^{(--)}\left(V_{2}\right)=-M
\end{array}
$$

where the matrix $M$ is defined by 8

$$
M_{r s}(\lambda)=\oint_{0} d z \oint_{0} d w z^{-r-\frac{1}{2}} w^{-s-\frac{1}{2}} \frac{1-\sqrt{\frac{w+\frac{1}{\lambda}}{z+\frac{1}{\lambda}}}}{z-w}=\frac{r}{r+s}\left(\begin{array}{c}
-\frac{1}{2} \\
r-\frac{1}{2}
\end{array}\right)\left(\begin{array}{c}
-\frac{1}{2} \\
s-\frac{1}{2}
\end{array}\right) \lambda^{r+s}
$$

An important consequence of the particular choice of emission points made in (33) is the very simple relation between $M^{(++)}\left(V_{1}\right)$ and $M^{(--)}\left(V_{2}\right)$; see (36) above (or (45) below), and as will be obvious later much of the analysis carried out in this paper relies heavily on this simple relation. We will return to the question of using arbitrary projective transformations in section 5. The other quantities in the vertex to be defined are the vectors $U_{r}^{( \pm)}$. They are given by

$$
\begin{aligned}
& \bar{U}_{R_{1}, r}^{(+)}=\bar{U}_{r m}^{(+)}\left(V_{1}\right) \hat{\bar{\psi}}_{m}^{R_{1}} \quad, \quad U_{R_{1}, r}^{(+)}=U_{r m}^{(+)}\left(V_{1}\right) \hat{\psi}_{m}^{R_{1}} \\
& \bar{U}_{R_{2}, r}^{(-)}=\bar{U}_{r m}^{(-)}\left(V_{2}\right) \hat{\bar{\psi}}_{m}^{R_{2}} \quad, \quad U_{R_{2}, r}^{(-)}=U_{r m}^{(-)}\left(V_{2}\right) \hat{\psi}_{m}^{R_{2}}
\end{aligned}
$$

For the above choices for $V_{1}$ and $V_{2}$ the matrices $U_{r m}^{( \pm)}\left(V_{i}\right)$ become

$$
\begin{array}{r}
U_{r m}^{(+)}\left(V_{1}\right)=\sqrt{2} v_{r}^{(m)} \\
U_{r m}^{(-)}\left(V_{2}\right)=-\sqrt{2} v_{r}^{(-m)}
\end{array}
$$

where we have defined

$$
v_{r}^{(m)}=\frac{1}{\sqrt{2}}\left(\begin{array}{c}
-m-\frac{1}{2} \\
r-\frac{1}{2}
\end{array}\right) \lambda^{m+r}
$$

Then by inserting coherent states between the reggeons 1 and 2, and performing the so obtained infinite dimensional integral one finds, for $\mathrm{d}$ complex fields, that

$$
\begin{array}{r}
\hat{W}_{R_{1} R_{2}}(\lambda)=\left[\operatorname{det}\left(1+M M^{T}\right)\right]^{d}: \exp \left(\bar{U}_{R_{2}}^{T(-)} \frac{1}{1+M^{T} M} U_{R_{1}}^{(+)}+U_{R_{2}}^{T(-)} \frac{1}{1+M M^{T}} \bar{U}_{R_{1}}^{(+)}\right. \\
\left.+U_{R_{2}}^{T(-)} M \frac{1}{1+M^{T} M} \bar{U}_{R_{2}}^{(-)}-\bar{U}_{R_{1}}^{T(+)} M \frac{1}{1+M^{T} M} U_{R_{1}}^{(+)}\right):
\end{array}
$$


We are now in the position where we can enumerate the quantities needed for the comparison of the above results to the proposed closed answer in the next section, and establish its correctness. Having already defined the infinite dimensional vectors $v^{(m)}$ the relevant quantities are

$$
X^{(m, n)}=v^{(m) T} \xi^{(n)}, \quad Y^{(m, n)}=v^{(m) T} \eta^{(n)},
$$

where

$$
\xi^{(m)}=\frac{1}{1+M} v^{(m)}, \quad \eta^{(m)}=\frac{1}{1-M} v^{(m)},
$$

which generalize quantities introduced in the early 1970's [8-17] (corresponding to $m=n=0$ of the above). In these references one may also find some of the tricks used below to manipulate these infinite dimensional matrices and vectors. The fact that all terms in the exponent of $\hat{W}_{R_{1} R_{2}}$ can be expressed in terms of $X^{(m, n)}$ and $Y^{(m, n)}$ can be seen as follows. First note that, writing $v$ instead of $v^{(0)}$,

$$
M_{r s}=H_{r s}+v_{r} v_{s}
$$

where $H$ is the antisymmetric part of $M$, and hence

$$
M^{T}=-M+2 v v^{T}
$$

Then the extremely useful matrix identity

$$
A^{-1}(A-B) B^{-1}=B^{-1}-A^{-1}
$$

with $A=1-M^{2}, B=1+M^{T} M=1-M^{2}+2 v v^{T} M$ gives

$$
\frac{1}{1+M^{T} M}=\frac{1}{1-M^{2}}-2 \frac{1}{1-M^{2}} v v^{T} M \frac{1}{1+M^{T} M}
$$

Multiplying this equation by $v^{T} M$ from the left produces an expression for $v^{T} M \frac{1}{1+M^{T} M}$ that can be inserted back into the last term on the right hand side of the last equation. Doing so, one finds

$$
\frac{1}{1+M^{T} M}=\frac{1}{1-M^{2}}-2 \frac{\frac{1}{1-M^{2}} v v^{T} \frac{M}{1-M^{2}}}{1+2 v^{T} \frac{M}{1-M^{2}} v}
$$


Finally, the identities

$$
\frac{1}{1-M^{2}}=\frac{1}{2}\left(\frac{1}{1-M}+\frac{1}{1+M}\right), \quad \frac{M}{1-M^{2}}=\frac{1}{2}\left(\frac{1}{1-M}-\frac{1}{1+M}\right)
$$

proves the assertion stated above.

Although we will later present explicit formulae only for those terms in the exponent of $\hat{W}_{R_{1} R_{2}}$ that are expressible in terms of $X^{(m, n)}$ and $Y^{(m, n)}$ with $m, n=0, \pm 1$, we emphasize here that it is possible to derive answers for all integers $m, n$. To explain how this can be done we divide the quantities $X^{(m, n)}$ and $Y^{(m, n)}$ into two groups; those with both $m$ and $n$ non-negative or with either $m$ or $n$ negative, and those with $m$ and $n$ both negative. The reason for this particular division will become clear below.

To deal with the first group we proceed as follows. As explained in detail elsewhere [11, 12, 38, it is possible to show that

$$
\xi_{r}=\frac{1}{\sqrt{2}} \oint_{C} \frac{d t}{2 \pi i t}\left(w_{\lambda}(t)\right)^{r}
$$

where

$$
w_{\lambda}(t)=\frac{t-\frac{(1+\lambda)^{2}}{4 \lambda}}{t(1-t)}
$$

and where the contour $C$ encircles the branch cut between $\mathrm{t}=0$ and $\mathrm{t}=1$, solves the equation

$$
(1+M) \xi=v
$$

and hence to conclude that $\xi$ is identical to what we above denoted as $\xi^{(0)}$. To prove that $\xi$ satisfies (52), one makes use of among other things the following change of variables

$$
t \mapsto \frac{(1+\lambda)^{2}}{4 \lambda t}
$$

which has the effect that

$$
w_{\lambda}(t) \mapsto \frac{1}{w_{\lambda}(t)}
$$

and that $C$ after the change encircles the cut between $t=\frac{(1+\lambda)^{2}}{4 \lambda}$ and $t=\infty$. Flipping the contour back to its original place gives the result that $\xi_{r}$ can also be written

$$
\xi_{r}=\frac{1}{\sqrt{2}} \oint_{C} \frac{d t}{2 \pi i t}\left(w_{\lambda}(t)\right)^{-r}
$$


(note the sign change in the exponent). Using either expression for $\xi_{r}$, one can easily do the sum in $v^{T} \xi=\sum_{r=\frac{1}{2}}^{\infty} v_{r} \xi_{r}$, and then perform a straightforward contour integral around two poles to find 12

$$
X^{(0,0)}=v^{T} \xi=\frac{1}{2}\left(1-\sqrt{\frac{1-\lambda}{1+\lambda}}\right)
$$

The corresponding formula for $\eta=\eta^{(0)}$ can be found from the $\lambda$-derivative of $\xi$. To get this relation, we define the matrix $R$ by

$$
R_{r s}=r \delta_{r s}
$$

and note that

$$
M R=R M^{T}
$$

From the definitions of $M$ and $v$ it follows that

$$
\lambda \frac{\partial}{\partial \lambda} M=2 R v v^{T}, \quad \lambda \frac{\partial}{\partial \lambda} v=R v
$$

which implies that

$$
\lambda \frac{\partial}{\partial \lambda} \frac{1}{1+M}=-\frac{1}{1+M}\left(\lambda \frac{\partial}{\partial \lambda} M\right) \frac{1}{1+M}=-2 \frac{1}{1+M} R v v^{T} \frac{1}{1+M}
$$

Using this equation when computing the $\lambda$-derivative of the equation $(1+M) \xi=v$ gives

$$
\lambda \frac{\partial}{\partial \lambda} \xi=R \tilde{\xi}\left(1-2 v^{T} \xi\right)
$$

where we have defined

$$
\tilde{\xi}=\frac{1}{1+M^{T}} v
$$

Next we define $\tilde{\eta}$ in an analogous fashion, and derive formulae relating them to their untilded counterparts by setting $A=1 \pm M^{T}$ and $B=1 \mp M$ in the matrix equation (46) and multiplying it by $v$ from the right. The result is

$$
\tilde{\xi}=\frac{\eta}{1+2 v^{T} \eta}, \quad \tilde{\eta}=\frac{\xi}{1-2 v^{T} \xi}
$$

for the upper and lower sign, respectively. Hence

$$
\lambda \frac{\partial}{\partial \lambda} \xi=R \eta \frac{1-2 v^{T} \xi}{1+2 v^{T} \eta}
$$


Multiplying also from the left with $v^{T}$ gives

$$
v^{T} \xi=\frac{v^{T} \eta}{1+2 v^{T} \eta}, \quad v^{T} \eta=\frac{v^{T} \xi}{1-2 v^{T} \xi}
$$

Thus eq. (56) implies that

$$
Y^{(0,0)}=v^{T} \eta=-\frac{1}{2}\left(1-\sqrt{\frac{1+\lambda}{1-\lambda}}\right)
$$

and, finally, from (64) we obtain

$$
\eta_{r}=\mp \frac{1}{\sqrt{2}} \oint_{C} \frac{d t}{2 \pi i t}\left(w_{\lambda}(t)\right)^{ \pm r} \frac{1}{1-\frac{4 \lambda t}{(1+\lambda)^{2}}}
$$

where $w_{\lambda}(t)$ and the contour $C$ are as defined for $\xi_{r}$ and where the two cases, differing by sign, are related through the same change of the variable $t$ as discussed above.

Note that, as previously found in [12],

$$
1-2 v^{T} \xi=\left(1+2 v^{T} \eta\right)^{-1}
$$

and that these quantities correspond to what in the bosonic case is denoted as $g^{\frac{1}{2}}$ in [21]. This relation is but a special case of the general relations between $X^{(m, n)}$ and $Y^{(m, n)}$ :

$$
\begin{aligned}
& Y^{(m, n)}=X^{(n, m)}+2 Y^{(m, 0)} X^{(n, 0)} \\
& X^{(m, n)}=Y^{(n, m)}-2 X^{(m, 0)} Y^{(n, 0)}
\end{aligned}
$$

derivable by using $A=1 \mp M$ and $B=1 \pm M^{T}$ in eq.46). However, comparing the explicit expressions (56) and (56) for $X^{(0,0)}$ and $Y^{(0,0)}$ above we discover a possible direct connection between them under $\lambda$ going to $-\lambda$. By expanding $\frac{1}{1 \pm M}$ in geometric series and comparing the power series in $\lambda$ of $X^{(m, n)}$ and $Y^{(m, n)}$ we discover immediately the powerful relation

$$
Y^{(m, n)}(\lambda)=(-1)^{m+n+1} X^{(m, n)}(-\lambda)
$$

valid for all integers $m, n$. This equation will be discussed again below.

To summarize, we have found explicit expressions for $\xi_{r}$ and $\eta_{r}$, and from these one can derive expressions for $\tilde{\xi}_{r}$ and $\tilde{\eta}_{r}$ as well as for $X^{(0,0)}$ and $Y^{(0,0)}$. In fact, in the same spirit as 
$X^{(0,0)}$ and $Y^{(0,0)}$ are derived from $\xi_{r}$ and $\eta_{r}$, one may also obtain closed answers for $X^{(m, 0)}$ and $Y^{(m, 0)}$ for $m$ any integer. By transposing and utilizing the formulae for $\tilde{\xi}_{r}$ and $\tilde{\eta}_{r}$ given above, also $X^{(0, m)}$ and $Y^{(0, m)}$ are easily found.

The quantities in the first group not yet discussed are the ones with both $m$ and $n \neq 0$. By transposing them if necessary these can always be written $X^{(m, n)}, Y^{(m, n)}$ with $n$ a positive integer. We now take advantage of the following property of the $v^{(n)}$ 's

$$
v^{(n+1)}=\lambda \frac{n+R}{n+\frac{1}{2}} v^{(n)}
$$

to step by step lower the superindex $n$ until it reaches $n=0$. The factors $\frac{n+R}{n+\frac{1}{2}}$ (recall that $R$ is the matrix defined in (57)) may then be brought through $\frac{1}{1 \pm M}$ using $\frac{1}{1 \pm M} R=R \frac{1}{1 \pm M^{T}}$ thereby producing a series of terms when hitting $v^{(m) T}$. Thus all $X^{(m, n)}, Y^{(m, n)}$ in the first group can be evaluated once $X^{(m, 0)}$ and $Y^{(m, 0)}$ are known.

Before turning to the second group of $X^{(m, n)}, Y^{(m, n)}$, i.e. those with both $m$ and $n$ negative integers, we apply the steps discussed above to arrive at the following expressions for the quantities in the first group (valid for the same $\lambda$ on the two sides of the equations)

$$
\begin{array}{ll}
X^{(1,1)}=\frac{3 Y^{(2,0)}-2 \lambda Y^{(1,0)}}{1+2 Y^{(0,0)}} & Y^{(1,1)}=\frac{3 X^{(2,0)}-2 \lambda X^{(1,0)}}{1-2 X^{(0,0)}} \\
X^{(0,1)}=\frac{Y^{(1,0)}}{1+2 Y^{(0,0)}} & Y^{(0,1)}=\frac{X^{(1,0)}}{1-2 X^{(0,0)}} \\
X^{(-1,1)}=\frac{2 \lambda Y^{(-1,0)}-4 Y^{(0,0)}}{1+2 Y^{(0,0)}} & Y^{(-1,1)}=\frac{2 \lambda X^{(-1,0)}-4 X^{(0,0)}}{1-2 X^{(0,0)}} \\
X^{(1,-1)}=Y^{(-1,1)}-\frac{2 Y^{(-1,0)}}{1+2 Y^{(0,0)}} Y^{(0,1)} & Y^{(1,-1)}=X^{(-1,1)}+\frac{2 X^{(-1,0)}}{1-2 X^{(0,0)}} X^{(0,1)} \\
X^{(0,-1)}=\frac{Y^{(-1,0)}}{1+2 Y^{(0,0)}} & Y^{(0,-1)}=\frac{X^{(-1,0)}}{1-2 X^{(0,0)}}
\end{array}
$$

where we should note that to evaluate $X^{(1,1)}$ we need $Y^{(2,0)}$ and similarly with $\mathrm{X}$ and $\mathrm{Y}$ interchanged.

When we now turn to the second group of $X^{(m, n)}, Y^{(m, n)}$ 's we will resort to different methods, since using (72) also here does not seem so convenient. More specifically, for these quantities we instead derive differential equations in $\lambda$. From (59) and (50) together with the relation

$$
\lambda \frac{\partial}{\partial \lambda} v^{(m)}=\frac{m+\frac{1}{2}}{\lambda} v^{(m+1)}
$$

we find that acting with the logarithmic $\lambda$-derivative on $X^{(m, n)}=v^{(m) T} \frac{1}{1+M} v^{(n)}$ gives

$$
\lambda \frac{\partial}{\partial \lambda} X^{(m, n)}=\frac{1}{\lambda}\left(\left(m+\frac{1}{2}\right) X^{(m+1, n)}+\left(n+\frac{1}{2}\right) X^{(m, n+1)}\right)
$$




$$
-2\left(\frac{m+\frac{1}{2}}{\lambda} Y^{(m+1,0)}-m Y^{(m, 0)}\right) \frac{Y^{(n, 0)}}{\left(1+2 Y^{(0,0)}\right)^{2}} \quad m, n \in \mathbf{Z}
$$

where we have also used the relations for $\tilde{\xi}_{r}$ and $\tilde{\eta}_{r}$ given in eq. (63). That equation (75) can be employed iteratively to obtain $X^{(m, n)}$ and $Y^{(m, n)}$ for arbitrary negative integers $m, n$ is clear from the form of its right hand side. Since, in this paper, we intend to give explicit results only for $m, n=0, \pm 1$ the last quantities to be computed are those for $m=n=-1$. This is accomplished by relating first $X^{(-1,-1)}$ to known quantities via (75), and then extract $Y^{(-1,-1)}$ from (69). Once again we find that it suffices to know $X^{(m, 0)}$ and $Y^{(m, 0)}$ for $m=-1,0,1,2$.

Let us now present the results for $X^{(m, 0)}$ and $Y^{(m, 0)}, m=-1,0,1,2$, derived from $\xi_{r}$ and $\eta_{r}$ by performing first the sums and then the contour integrals in $v^{(m) T} \xi$ and $v^{(m) T} \eta$. After some computation one finds the expressions presented in table 1.

$$
\begin{array}{lll}
m & X^{(m, 0)} & Y^{(m, 0)} \\
2 & \frac{\lambda^{3}}{2} \frac{1-\lambda}{\left(1-\lambda^{2}\right)^{5 / 2}}\left(1+\frac{\lambda}{2}-\frac{\lambda^{2}}{2}-\frac{3}{8} \lambda^{3}\right) & \frac{\lambda^{3}}{2} \frac{1+\lambda}{\left(1-\lambda^{2}\right)^{5 / 2}}\left(1-\frac{\lambda}{2}-\frac{\lambda^{2}}{2}+\frac{3}{8} \lambda^{3}\right) \\
1 & \frac{\lambda^{2}}{2} \frac{1}{1+\lambda} \frac{1}{\sqrt{1-\lambda^{2}}}\left(1+\frac{\lambda}{2}\right) & \frac{\lambda^{2}}{2} \frac{1}{1-\lambda} \frac{1}{\sqrt{1-\lambda^{2}}}\left(1-\frac{\lambda}{2}\right) \\
0 & \frac{1}{2}\left(1-\sqrt{\frac{1-\lambda}{1+\lambda}}\right) & -\frac{1}{2}\left(1-\sqrt{\frac{1+\lambda}{1-\lambda}}\right) \\
-1 & \frac{1}{2}\left(1-\frac{\lambda}{2}\right) & \frac{1}{2}\left(1+\frac{\lambda}{2}\right)
\end{array}
$$

Table 1: $X^{(m, 0)}=v^{(m) T} \xi$ and $Y^{(m, 0)}=v^{(m) T} \eta$ for $m=-1,0,1,2$.

Inserting the above results into eqs. $(\sqrt{73})$ and $(\sqrt{75})$ we get the remaining quantities of interest as given in table 2 .

$$
\begin{array}{lll}
(m, n) & X^{(m, n)} & Y^{(m, n)} \\
(1,1) & \frac{\lambda^{3}}{2} \frac{1}{\left(1-\lambda^{2}\right)^{2}}\left(1-\frac{\lambda}{2}+\frac{\lambda^{2}}{2}+\frac{\lambda^{3}}{8}\right) & \frac{\lambda^{3}}{2} \frac{1}{\left(1-\lambda^{2}\right)^{2}}\left(1+\frac{\lambda}{2}+\frac{\lambda^{2}}{2}-\frac{\lambda^{3}}{8}\right) \\
(0,1) & \frac{\lambda}{2} \frac{1-\frac{\lambda}{2}}{1-\lambda^{2}} & -\frac{\lambda}{2} \frac{1+\frac{\lambda}{2}}{1-\lambda^{2}} \\
(-1,1) & \frac{1}{2}\left((1+\lambda) \sqrt{1-\lambda^{2}}-1\right) & -\frac{1}{2}\left((1-\lambda) \sqrt{1-\lambda^{2}}-1\right) \\
(1,-1) & \frac{1}{2}\left(1-\frac{1}{1+\lambda} \frac{1}{\sqrt{1-\lambda^{2}}}\left(1-\lambda^{2}+\lambda^{3}+\frac{5 \lambda^{4}}{4}\right)\right) & -\frac{1}{2}\left(1-\frac{1}{1-\lambda} \frac{1}{\sqrt{1-\lambda^{2}}}\left(1-\lambda^{2}-\lambda^{3}+\frac{5 \lambda^{4}}{4}\right)\right) \\
(0,-1) & \frac{1}{2} \sqrt{\frac{1-\lambda}{1+\lambda}}\left(1+\frac{\lambda}{2}\right) & -\frac{1}{2} \sqrt{\frac{1+\lambda}{1-\lambda}}\left(1-\frac{\lambda}{2}\right) \\
(-1,-1) & \frac{1}{2 \lambda}\left(1-\frac{\lambda}{2}+\frac{\lambda^{2}}{2}+\frac{\lambda^{3}}{8}\right) & \frac{1}{2 \lambda}\left(1+\frac{\lambda}{2}+\frac{\lambda^{2}}{2}-\frac{\lambda^{3}}{8}\right)
\end{array}
$$

Table $2: X^{(m, n)}=v^{(m) T} \frac{1}{1+M} v^{(n)}$ and $Y^{(m, n)}=v^{(m) T} \frac{1}{1-M} v^{(n)}$ for $m, n=-1,0,1$, except those listed in table 1. 
It is gratifying to verify the relation (17) between the X's and the Y's obtained by letting $\lambda$ go to $-\lambda$. Strictly speaking, of course, having established this relation independent computations of the X's and Y's from $\xi$ and $\eta$ are not necessary and may be viewed as a mere check on the formalism.

Finally, as explained in the beginning of this section, it is now a simple matter to derive the expressions appearing in the exponent of the vertex $\hat{W}_{R_{1} R_{2}}$. The ones we promised to present the answers for are given in table 3 , where we have not tabulated $v^{(m) T} M^{T} \frac{1}{1+M M^{T}} v^{(n)}$ since it can be seen to equal $v^{(n) T} M \frac{1}{1+M^{T} M} v^{(m)}$.

$$
\begin{array}{llll}
(m, n) & v^{(m) T} \frac{1}{1+M^{T} M} v^{(n)} & v^{(m) T} \frac{1}{1+M M^{T}} v^{(n)} & v^{(m) T} M \frac{1}{1+M^{T} M} v^{(n)} \\
(1,1) & \frac{\lambda^{3}}{2} \frac{1}{\left(1-\lambda^{2}\right)^{2}}\left(1+\frac{\lambda^{4}}{4}\right) & \frac{\lambda^{3}}{2} \frac{1}{\left(1-\lambda^{2}\right)^{2}} & \frac{\lambda^{4}}{4} \frac{1}{\left(1-\lambda^{2}\right)^{2}}\left(1-\frac{3}{4} \lambda^{2}\right) \\
(1,0) & \frac{\lambda^{2}}{2} \frac{1}{\left(1-\lambda^{2}\right)}\left(1-\frac{\lambda^{2}}{2}\right) & \frac{\lambda^{2}}{2} \frac{1}{\sqrt{1-\lambda^{2}}} & \frac{\lambda^{3}}{4} \frac{1}{\left(1-\lambda^{2}\right)} \\
(0,1) & \frac{\lambda^{2}}{2} \frac{1}{\left(1-\lambda^{2}\right)}\left(1-\frac{\lambda^{2}}{2}\right) & \frac{\lambda^{2}}{2} \frac{1}{\sqrt{1-\lambda^{2}}} & \frac{\lambda^{3}}{4} \frac{1}{\sqrt{1-\lambda^{2}}} \\
(-1,1) & \frac{\lambda}{2} \frac{1}{\sqrt{1-\lambda^{2}}}\left(1-\frac{3}{2} \lambda^{2}\right) & \frac{\lambda}{2} \frac{1}{\sqrt{1-\lambda^{2}}}\left(1-\frac{3}{2} \lambda^{2}\right) & \frac{1}{2}\left(1-\frac{1}{\sqrt{1-\lambda^{2}}}\left(1-\frac{\lambda^{2}}{2}\right)^{2}\right) \\
(0,0) & \frac{\lambda}{2} & \frac{\lambda}{2} & \frac{1}{2}\left(1-\sqrt{1-\lambda^{2}}\right) \\
(1,-1) & \frac{\lambda}{2} \frac{1}{\sqrt{1-\lambda^{2}}}\left(1-\frac{3}{2} \lambda^{2}\right) & \frac{\lambda}{2} \frac{1}{\sqrt{1-\lambda^{2}}}\left(1-\frac{3}{2} \lambda^{2}\right) & \frac{1}{2}\left(\frac{1}{\sqrt{1-\lambda^{2}}}-1\right) \\
(-1,0) & \frac{1}{2} \sqrt{1-\lambda^{2}} & \frac{1}{2}\left(1-\frac{\lambda^{2}}{2}\right) & \frac{\lambda}{4} \sqrt{1-\lambda^{2}} \\
(0,-1) & \frac{1}{2} \sqrt{1-\lambda^{2}} & \frac{1}{2}\left(1-\frac{\lambda^{2}}{2}\right) & \frac{\lambda}{4} \\
(-1,-1) & \frac{1}{2 \lambda} & \frac{1}{2 \lambda}\left(1+\frac{\lambda^{4}}{4}\right) & \frac{1}{4}\left(1-\frac{3 \lambda^{4}}{4}\right)
\end{array}
$$

Table 3 : Quantities appearing in the exponent of $\hat{W}_{R_{1} R_{2}}$.

These expressions are interesting also for the reason that they seem, at least in some cases, to relate to generating functions for Catalan numbers which appear frequently in combinatorics and statistical mechanics problems. This observation was made in [38] where the equation

$$
v^{T} \frac{1}{1-H^{2}} v=\frac{1}{\lambda}\left(1-\sqrt{1-\lambda^{2}}\right)
$$

(recall that $H$ is the antisymmetric part of the matrix $M$ ) is discussed and used to derive a new highly non-linear relation between binary Catalan numbers. The connection to the Catalan 
numbers $c_{k}$ stems from the fact that their generating function,

$$
S(x)=\frac{1-\sqrt{1-4 x}}{2 x}=\sum_{k=0}^{\infty} c_{k} x^{k}
$$

obeys the algebraic equation

$$
x S^{2}-S+1=0
$$

From this ones concludes that $c_{0}=1$ and

$$
c_{k}=\frac{1}{k+1}\left(\begin{array}{c}
2 k \\
k
\end{array}\right) \quad, \quad k \geq 1
$$

It is not clear to us what the combinatorial reason behind the occurence of Catalan numbers in this context is, and it would probably be worthwhile to try to gain some understanding of Ramond vertices from this point of view. For the benefit of the reader we quote here the non-linear relation found in [38]:

$$
c_{k}=\sum_{m=0}^{\left[\frac{k}{2}\right]} 2^{2(m-k)} \sum_{n_{\mu}}\left(\prod_{\mu=1}^{2 m} \frac{n_{\mu-1}-n_{\mu}}{n_{\mu-1}+n_{\mu}+1}\right)\left(\prod_{\mu=0}^{2 m}\left(n_{\mu}+1\right) c_{n_{\mu}}\right)^{2}
$$

where $n_{\mu} \in \mathbf{Z}_{+}$are restricted by $\sum_{\mu=0}^{2 m} n_{\mu}=k-m$ and where $[x]$ denotes the integer part of $x$.

We conclude this section by briefly explaining how to arrive at an answer for $\operatorname{det}\left(1+M^{T} M\right)$. First we relate it to $\operatorname{det}\left(1-M^{2}\right)$ which was computed in [12]. The relation between these two determinants can be obtained by applying the same trick as was used in [12 to derive a relation between $\operatorname{det}\left(1-H^{2}\right)$ and $\operatorname{det}\left(1-M^{2}\right)$. First note that eq. (45) implies that $1+M^{T} M=$ $1-M^{2}+2 v v^{T} M$. If we divide this equation by $1-M^{2}$ and then take the determinant of it we get

$$
\operatorname{det}\left(1+M^{T} M\right)=\left(1+2 v^{T} \frac{M}{1-M^{2}} v\right) \operatorname{det}\left(1-M^{2}\right)
$$

Using the results in 12 we can thus summarize the answers for these determinants as follows

$$
\begin{aligned}
\operatorname{det}\left(1+M^{T} M\right) & =\left(1-\lambda^{2}\right)^{-\frac{1}{4}}=\operatorname{det}\left(1+M M^{T}\right) \\
\operatorname{det}\left(1-M^{2}\right) & =\left(1-\lambda^{2}\right)^{\frac{1}{4}} \\
\operatorname{det}\left(1-H^{2}\right) & =\frac{1}{2}\left(\left(1-\lambda^{2}\right)^{-\frac{1}{4}}+\left(1-\lambda^{2}\right)^{\frac{1}{4}}\right)
\end{aligned}
$$

We will have reason to return to these equations in the next section. 


\section{The closed form of the complex four-Ramond reggeon vertex}

The purpose of this section is to propose a closed form of the four-Ramond reggeon vertex discussed from the point of view of sewing in the previous sections of this paper. As will be obvious below, this closed form reproduces all our explicit results obtained in section 3 in a very direct manner. It has a quite natural form dictated by the structure of branch cuts generated by the twisted fields present, and reads (for $d=1$ )

$$
\begin{aligned}
& \hat{W}_{R_{1} R_{2}}(\lambda)=\left\langle 0\left|: \hat{\bar{S}}(\infty) \hat{S}\left(-\frac{1}{\lambda}\right):: \hat{\bar{S}}(-\lambda) \hat{S}(0):\right| 0\right\rangle \times \\
& : \exp \oint_{C_{z}} d z \oint_{C_{w}} d w \hat{\bar{\psi}}_{R}^{V^{-1}}(z) \frac{\left\langle 0\left|\hat{\psi}(z) \hat{\bar{\psi}}(w): \hat{\bar{S}}(\infty) \hat{S}\left(-\frac{1}{\lambda}\right):: \hat{\bar{S}}(-\lambda) \hat{S}(0):\right| 0\right\rangle}{\left\langle 0\left|: \hat{\bar{S}}(\infty) \hat{S}\left(-\frac{1}{\lambda}\right):: \hat{\bar{S}}(-\lambda) \hat{S}(0):\right| 0\right\rangle} \hat{\psi}_{R}^{V^{-1}}(w):
\end{aligned}
$$

where radial ordering of the operators is understood in all correlation functions. The $\hat{\psi}_{R}^{V^{-1}}$ 's appearing in the exponent are sums of the two external twisted fields transformed with their respective projective transformation. The contours $C_{z}$ and $C_{w}$ enclose all branch cuts appearing

in $\hat{\bar{\psi}}_{R}^{V^{-1}}(z)$ and $\hat{\psi}_{R}^{V^{-1}}(w)$, respectively. The normal ordering outside the exponential refers only to these fields, while the normal ordering (also denoted by double dots) inside the correlation functions refers to the chiral boson in terms of which all spin fields $\hat{S}(z), \hat{\bar{S}}(z)$, and fermion fields $\hat{\bar{\psi}}(z), \hat{\psi}(z)$ are bosonized. We have chosen to normal order separately each pair of spin fields related to the same vertex, but it should be clear from the correlators in the exponent that the denominator can be eliminated simply by the normal ordering of all four spin fields in the numerator. On the other hand, one also easily sees that by keeping the denominator one can in fact remove all normal ordering dots in the correlation functions in the exponent of (87).

The exponential factor of this vertex gives, as is readily verified (see below), exactly the results quoted in table 3 for all terms containing only twisted zero and/or level one modes, while the prefactor correlation function reproduces the determinant in equation (84). There can be hardly any doubt that this will extend to all higher modes, and we have therefore managed to show, without providing a completely rigorous proof, that the vertex given above (in this section) is the correct answer for the sewed vertex with four external $R$ legs. We will come 
back to the issue of a rigorous proof in a future publication.

The form of the vertex given here, i.e. in terms of correlation functions of spin fields etc, was suggested by LeClair in [36] for the case of one pair of twisted $(R)$ external fields (one branch cut) and one untwisted $(N S)$ one, while the generalization to an arbitrary number of $N S$ and $R$ external legs was suggested in [37] based on path integral arguments, which, however, cannot in any obvious way be transferred into the operator methods used in this paper. In ref. [37] the authors also performed the infinite dimensional integrals giving the NSR vertex corresponding to our eq. (41) (see also the next section where our version of their result is given and the differences explained) but the check of the closed form was restricted to the one term in the exponent containing only zero modes. This term is a special combination of $X^{(0,0)}$ and $Y^{(0,0)}$ (see section 3$)$ and equals the entry for $(m, n)=(0,0)$ in table 5 . The work presented in section 3 then essentially extends these old results to all terms in the exponent by giving an algorithm for how they may be computed, and with explicit expressions presented for all terms containing only zero and/or level one modes.

To get a feeling for the closed form of the reggeon vertex given in (87), let us evaluate the correlation function in the exponent:

$$
\begin{aligned}
& \frac{\left\langle 0\left|\hat{\psi}(z) \hat{\bar{\psi}}(w): \hat{\bar{S}}(\infty) \hat{S}\left(-\frac{1}{\lambda}\right):: \hat{\bar{S}}(-\lambda) \hat{S}(0):\right| 0\right\rangle}{\left\langle 0\left|: \hat{\bar{S}}(\infty) \hat{S}\left(-\frac{1}{\lambda}\right):: \hat{\bar{S}}(-\lambda) \hat{S}(0):\right| 0\right\rangle}= \\
& =\left\langle 0\left|\hat{\psi}(z) \hat{\bar{\psi}}(w): \hat{\bar{S}}(\infty) \hat{S}\left(-\frac{1}{\lambda}\right) \hat{\bar{S}}(-\lambda) \hat{S}(0):\right| 0\right\rangle=\frac{\sqrt{\frac{z+\frac{1}{\lambda}}{w+\frac{1}{\lambda}} \frac{z}{\lambda}+1} \frac{\frac{w}{\lambda}+1}{w}}{z-w}
\end{aligned}
$$

The integrals in the exponent of (87) are then trivial to perform because the Ramond fields multiplying the square root contain half integer powers of the very same factors that appear under the square root, and hence the contours $C_{z}$ and $C_{w}$ encircle in fact only poles and no branch cuts. If external $N S$ legs are added to the surface, one therefore has to keep their emission points away from the branch points. In the last section, we will return to the question of how the above form of the vertex can be generalized to an arbitrary number of external $R$ as well as $N S$ legs.

If one rewrites equation (88) and the determinant prefactor using the projective transfor- 
mations $V_{i}^{-1}$ then one finds the following alternative closed form of the four-Ramond vertex:

$$
\hat{W}_{R_{1} R_{2}}\left(V_{1}, V_{2}\right)=\left(\frac{V_{2}^{-1}\left(z_{1}^{(1)}\right)}{V_{2}^{-1}\left(z_{2}^{(1)}\right)}\right)^{\frac{1}{4}}: \exp \left(\oint_{C_{z}} d z \oint_{C_{w}} d w \hat{\psi}_{R}^{V^{-1}}(z) G\left(V_{1}, V_{2} ; z, w\right) \hat{\bar{\psi}}_{R}^{V^{-1}}(w)\right):
$$

where the propagator $G\left(V_{1}, V_{2} ; z, w\right)$ is given by

$$
G\left(V_{1}, V_{2} ; z, w\right)=\frac{\sqrt{\frac{V_{1}^{-1}(w) V_{2}^{-1}(w)}{V_{1}^{-1}(z) V_{2}^{-1}(z)}}}{z-w}
$$

This propagator may be compared to $M\left(z_{1}, z_{2} ; z, w\right)$ appearing in the exponent of dual twisted vertex $\hat{W}_{R}(V)$ (see eqs. (19),(23), (24)) which is actually not a propagator since it is regular at $z=w$. Nevertheless, these two objects are so similar that it must be considered rather strange that a simple way of relating them does not seem to exist. Note that in the prefactor the role of the two projective transformations can be interchanged without altering the result. As we will see in the next section using other choices of projective transformations in the sewing calculation will give rise to closed forms of the four-vertex which if written in terms of spin fields differ from the one presented in the beginning of this section. Not surprisingly, however, the form given in (89) is universal to all cases (i.e. choices of projective transformations) studied here.

\section{General forms of the four-vertex for real and complex fields}

In this section we will discuss, for both real and complex fields, the dependence of the sewed four-vertex on the choice of projective transformations. When we described in the beginning of section 3 how to derive the four-reggeon vertex for complex fermions by sewing we picked two specific projective transformations $V_{1}$ and $V_{2}$. These may, however, be chosen arbitrarily as long as they together contain only one moduli parameter. Here we will restrict our discussion to three other related choices corresponding to replacing either $V_{1}, V_{2}$ or both by $V_{1} \Gamma$ and $V_{2} \Gamma$, respectively, where $\Gamma(z)=1 / z$. We do not attempt, however, to perform the sewing with more general projective transformations than $V_{1}$ and $V_{2}$ since it seems that many of the tricks used 
in section 3 are then no longer applicable. This is due to the fact that they depended on the close resemblance between the matrices $M^{( \pm \pm)}\left(V_{i}\right)$ that results from the choices of $V_{1}$ and $V_{2}$ made here.

For these new choices the corresponding results from sewing are all of the same form as the one found in the previous section, and the closed forms differ only by moving around the bar on the bosonized spin fields in the correlation functions appearing in (87). We summarize this as follows:

$$
\begin{aligned}
& \hat{W}_{R_{1}, R_{2}}(\lambda)=\operatorname{det}\left(1+M^{(++)}\left(V_{1}\right) M^{(--)}\left(V_{2}\right)\right): \exp \left(\sum_{i, j=1,2} \sum_{m, n} \hat{\psi}_{m}^{R_{i}} A_{m n}^{i j}(\lambda) \hat{\bar{\psi}}_{n}^{R_{j}}\right):= \\
& =\left\langle 0\left|: \bar{S}\left(z_{2}^{(1)}\right) S\left(z_{1}^{(1)}\right):: \bar{S}\left(z_{2}^{(2)}\right) S\left(z_{2}^{(1)}\right):\right| 0\right\rangle \\
& : \exp \left(\oint_{C_{z}} d z \oint_{C_{w}} d w \hat{\bar{\psi}}_{R}^{V^{-1}}(z)\left\langle 0\left|\psi(z) \bar{\psi}(w): \bar{S}\left(z_{2}^{(1)}\right) S\left(z_{1}^{(1)}\right) \bar{S}\left(z_{2}^{(2)}\right) S\left(z_{1}^{(2)}\right):\right| 0\right\rangle \hat{\psi}_{R}^{V^{-1}}(w)\right):
\end{aligned}
$$

where we have defined the infinite dimensional matrices $A^{i j}$ through

$$
\begin{aligned}
A_{m n}^{11} & =\sum_{r, s} U_{r m}^{(+)}\left(V_{1}\right)\left[M^{(--)}\left(V_{2}\right) \frac{1}{1+M^{(++)}\left(V_{1}\right) M^{(--)}\left(V_{2}\right)}\right]_{r s} \bar{U}_{s n}^{(+)}\left(V_{1}\right) \\
A_{m n}^{22} & =\sum_{r, s} U_{r m}^{(-)}\left(V_{2}\right)\left[M^{(++)}\left(V_{1}\right) \frac{1}{1+M^{(--)}\left(V_{2}\right) M^{(++)}\left(V_{1}\right)}\right]_{r s} \bar{U}_{s n}^{(-)}\left(V_{2}\right) \\
A_{m n}^{12} & =-\sum_{r, s} U_{r m}^{(+)}\left(V_{1}\right)\left[\frac{1}{1+M^{(--)}\left(V_{2}\right) M^{(++)}\left(V_{1}\right)}\right]_{r s} \bar{U}_{s n}^{(-)}\left(V_{2}\right) \\
A_{m n}^{21} & =\sum_{r, s} U_{r m}^{(-)}\left(V_{2}\right)\left[\frac{1}{1+M^{(++)}\left(V_{1}\right) M^{(--)}\left(V_{2}\right)}\right]_{r s} \bar{U}_{s n}^{(+)}\left(V_{1}\right)
\end{aligned}
$$


and where the four cases discussed above are given by

$$
\begin{aligned}
& z_{1}^{(1)}, z_{2}^{(1)} \quad V_{1}^{-1}(z)=a \frac{z-z_{1}^{(1)}}{z-z_{2}^{(1)}} \quad U_{r m}^{(+)}\left(V_{1}\right)=\bar{U}^{(+)}\left(V_{1}\right) \quad M^{(++)}\left(V_{1}\right) \\
& z_{1}^{(2)}, z_{2}^{(2)} \quad V_{2}^{-1}(z)=a \frac{z-z_{1}^{(2)}}{z-z_{2}^{(2)}} \quad U_{r m}^{(-)}\left(V_{2}\right)=\bar{U}^{(-)}\left(V_{2}\right) \quad M^{(--)}\left(V_{2}\right) \\
& \begin{array}{lllll}
I & -\frac{1}{\lambda}, \infty & z+\frac{1}{\lambda} & & \\
2 & v_{r}^{(m)}(\lambda) & M(\lambda)
\end{array} \\
& \begin{array}{cccc}
0,-\lambda & \frac{z}{\lambda} & -\sqrt{2} v_{r}^{(-m)}(\lambda) & M^{T}(\lambda)
\end{array} \\
& \text { II } \infty,-\frac{1}{\lambda} \quad \frac{1}{z+\frac{1}{\lambda}} \quad \sqrt{2} i v_{r}^{(-m)}(\lambda) \quad-M^{T}(\lambda) \\
& \begin{array}{llll}
-\lambda, 0 & \frac{z}{\lambda}+1 & -\sqrt{2} i v_{r}^{(m)}(\lambda) & -M(\lambda)
\end{array} \\
& \begin{array}{llll}
I I I-\frac{1}{\lambda}, \infty & z+\frac{1}{\lambda} & \sqrt{2} v_{r}^{(m)}(\lambda) & M(\lambda)
\end{array} \\
& \begin{array}{llll}
-\lambda, 0 & \frac{z}{\lambda}+1 & -\sqrt{2} i v_{r}^{(m)}(\lambda) & -M(\lambda)
\end{array} \\
& \text { IV } \infty,-\frac{1}{\lambda} \quad \frac{1}{z+\frac{1}{\lambda}} \quad \sqrt{2} i v_{r}^{(-m)}(\lambda) \quad-M^{T}(\lambda) \\
& \begin{array}{llll}
0,-\lambda & \frac{z}{z}+1 & -\sqrt{2} v_{r}^{(-m)}(\lambda) & M^{T}(\lambda)
\end{array}
\end{aligned}
$$

For cases $I I I$ and $I V$ the results of the calculation of the matrix elements at level 0 and 1 in (91) using the sewing methods described in section three are collected in table 4 .

The four complex situations described above can still be summarized in one general closed formula for the vertex, namely (89), the only difference being the choice of projective transformations made in these cases. 


$$
\begin{array}{lll}
(m, n) & v^{(m) T} \frac{M}{1-M^{2}} v^{(n)} & v^{(m) T} \frac{1}{1-M^{2}} v^{(n)} \\
(1,1) & \frac{\lambda^{4}}{4} \frac{1}{\left(1-\lambda^{2}\right)^{2}}\left(1-\frac{\lambda^{2}}{4}\right) & \frac{\lambda^{3}}{2} \frac{1}{\left(1-\lambda^{2}\right)^{2}}\left(1+\frac{\lambda^{2}}{2}\right) \\
(1,0) & \frac{\lambda^{3}}{4} \frac{1}{\left(1-\lambda^{2}\right)^{\frac{3}{2}}} & \frac{\lambda^{2}}{2} \frac{1}{\left(1-\lambda^{2}\right)^{\frac{3}{2}}}\left(1-\frac{\lambda^{2}}{2}\right) \\
(0,1) & \frac{\lambda^{3}}{4} \frac{1}{\left(1-\lambda^{2}\right)} & \frac{\lambda^{2}}{2} \frac{1}{\left(1-\lambda^{2}\right)} \\
(-1,1) & \frac{1}{2}\left(1-\sqrt{1-\lambda^{2}}\right) & \frac{\lambda}{2} \sqrt{1-\lambda^{2}} \\
(0,0) & \frac{1}{4}\left(\sqrt{\frac{1+\lambda}{1-\lambda}}+\sqrt{\frac{1-\lambda}{1+\lambda}}-2\right) & \frac{1}{4}\left(\sqrt{\frac{1+\lambda}{1-\lambda}}-\sqrt{\frac{1-\lambda}{1+\lambda}}\right) \\
(1,-1) & \frac{1}{2}\left(\frac{1}{\left(1-\lambda^{2}\right)^{\frac{3}{2}}}\left(1-\frac{\lambda^{2}}{2}\right)^{2}-1\right) & \frac{\lambda}{2} \frac{1}{\left(1-\lambda^{2}\right)^{\frac{3}{2}}}\left(1-2 \lambda^{2}+\frac{5 \lambda^{4}}{4}\right) \\
(-1,0) & \frac{\lambda}{4} & \frac{1}{2} \\
(0,-1) & \frac{\lambda}{4} \frac{1}{\sqrt{1-\lambda^{2}}} & \frac{1}{2} \frac{1}{\sqrt{1-\lambda^{2}}}\left(1-\frac{\lambda^{2}}{2}\right) \\
(-1,-1) & \frac{1}{4}-\frac{\lambda^{2}}{16} & \frac{1}{2 \lambda}+\frac{\lambda}{4}
\end{array}
$$

Table 4: Level 0 and 1 matrix elements in the complex cases $I I I$ and $I V$.

Having derived these complex four-vertices it is now easy to write down the answer also for the case of real fermions taking into account the fact that, as is usually done, the zero mode is not included in the normal ordering (for $D$ real fields):

$$
\begin{aligned}
& \hat{W}_{R_{1}, R_{2}}= \\
& =\operatorname{det}\left(1-H^{2}\right)^{\frac{D}{2}} \times
\end{aligned}
$$

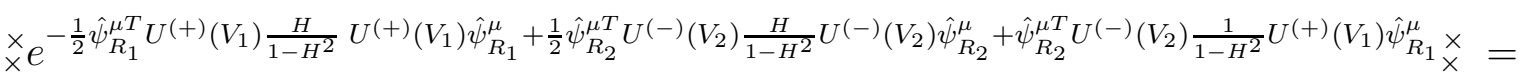

$$
\begin{aligned}
& =\left\langle 0\left|:(\hat{\bar{S}} \hat{S})\left(\infty,-\frac{1}{\lambda}\right)::(\hat{\bar{S}} \hat{S})(-\lambda, 0):\right| 0\right\rangle^{\frac{D}{2}} \times \\
& \stackrel{\times}{\times} \exp \left(\frac{1}{2} \oint_{C_{z}} d z \oint_{C_{w}} \hat{\psi}_{R}^{\mu V^{-1}}(z) \frac{\left\langle 0\left|(\hat{\psi} \hat{\psi})(z, w):(\hat{S} \hat{S})\left(\infty,-\frac{1}{\lambda}\right)::(\hat{S} \hat{S})(-\lambda, 0):\right| 0\right\rangle}{\left\langle 0\left|:(\hat{S} \hat{S})\left(\infty,-\frac{1}{\lambda}\right)::(\hat{S} \hat{S})(-\lambda, 0):\right| 0\right\rangle} \hat{\psi}_{R}^{\mu V^{-1}}(w)\right)_{\times}^{\times}= \\
& =\left(\frac{\left(\frac{V_{2}^{-1}\left(z_{1}^{(1)}\right)}{V_{2}^{-1}\left(z_{2}^{(1)}\right)}\right)^{\frac{1}{4}}+\left(\frac{V_{2}^{-1}\left(z_{2}^{(1)}\right)}{V_{2}^{-1}\left(z_{1}^{(1)}\right)}\right)^{\frac{1}{4}}}{2}\right)^{\frac{D}{2}} \times \\
& \stackrel{\times}{\times} \exp \left(\frac{1}{2} \oint_{C_{z}} d z \oint_{C_{w}} d w \hat{\psi}_{R}^{\mu V^{-1}}(z) K\left(V_{1}, V_{2} ; z, w\right) \hat{\psi}_{R}^{\mu V^{-1}}(w)\right) \times
\end{aligned}
$$

The results from the sewing are given in equation (96), where $H=\frac{1}{2}\left(M-M^{T}\right)$ and where $U^{( \pm)}$ is defined in (31). The explicit result for the determinant is given in (86), while the explicit results for level 0 and 1 calculated using the method explained in section three are collected in table 5. The closed forms, which are given in equations (97) and (98), are obtained by 
substituting in (87) each pair of spin fields by their symmetric combinations, i.e. instead of $\left.\hat{\bar{S}}\left(z_{2}^{(i)}\right)\right) \hat{S}\left(z_{1}^{(i)}\right)$ we use, as already noticed in 37,

$$
(\hat{\bar{S}} \hat{S})\left(z_{2}^{(i)}, z_{1}^{(i)}\right)=\frac{1}{2}\left(\hat{\bar{S}}\left(z_{2}^{(i)}\right) \hat{S}\left(z_{1}^{(i)}\right)+\hat{S}\left(z_{2}^{(i)}\right) \hat{\bar{S}}\left(z_{1}^{(i)}\right)\right)
$$

The propagator in this case becomes

$$
K\left(V_{1}, V_{2} ; z, w\right)=\frac{1}{2} \frac{1}{z-w} \frac{\left(\frac{V_{2}^{-1}\left(z_{1}^{(1)}\right)}{V_{2}^{-1}\left(z_{2}^{(1)}\right)}\right)^{\frac{1}{4}} \sqrt{\frac{V_{1}^{-1}(z)}{V_{1}^{-1}(w)} \frac{V_{2}^{-1}(z)}{V_{2}^{-1}(w)}}+\left(\frac{V_{2}^{-1}\left(z_{2}^{(1)}\right)}{V_{2}^{-1}\left(z_{1}^{(1)}\right)}\right)^{\frac{1}{4}} \sqrt{\frac{V_{1}^{-1}(w)}{V_{1}^{-1}(z)} \frac{V_{2}^{-1}(z)}{V_{2}^{-1}(w)}}+(z \leftrightarrow w)}{\left(\frac{V_{2}^{-1}\left(z_{1}^{(1)}\right)}{V_{2}^{-1}\left(z_{2}^{(1)}\right)}\right)^{\frac{1}{4}}+\left(\frac{V_{2}^{-1}\left(z_{2}^{(1)}\right)}{V_{2}^{-1}\left(z_{1}^{(1)}\right)}\right)^{\frac{1}{4}}}
$$

Note that unlike the complex case the denominator in the exponent of (97) does not cancel the correlations between the spin fields in the numerator. Another difference to the complex case is that the propagators in (96), (97) and (98) are the same in all four cases in (94), since under the interchanges $z_{1}^{(i)} \leftrightarrow z_{2}^{(i)}$ the matrices $H^{( \pm \pm)}\left(z_{1}^{(i)}, z_{2}^{(i)}\right)$ and the symmetric combinations $(\hat{S} \hat{S})\left(z_{1}^{(i)}, z_{2}^{(i)}\right)$ are invariant.

$$
\begin{array}{lll}
(m, n) & v^{(m) T} \frac{1}{1-H^{2}} H v^{(n)} & v^{(m) T} \frac{1}{1-H^{2}} v^{(n)} \\
(1,1) & 0 & \frac{\lambda^{3}}{4} \frac{1}{\left(1-\lambda^{2}\right)^{2}}\left[1+\frac{5 \lambda^{2}}{4}+\sqrt{1-\lambda^{2}}\left(1-\frac{\lambda^{4}}{4}\right)\right] \\
(0,1) & -\frac{\lambda}{4} \frac{1}{1-\lambda^{2}}\left(1-\sqrt{1-\lambda^{2}}\right)^{2} & \frac{\lambda^{2}}{4} \frac{1}{\left(1-\lambda^{2}\right)}\left[1+\sqrt{1-\lambda^{2}}\right] \\
(-1,1) & \frac{1}{2} \frac{1}{\left(1-\lambda^{2}\right)}\left[1-\lambda^{2}-\frac{\lambda^{4}}{8}-\sqrt{1-\lambda^{2}}\left(1-\frac{\lambda^{2}}{2}\right)\right] & \frac{\lambda}{4} \frac{1}{\left(1-\lambda^{2}\right)}\left[1-\frac{3 \lambda^{2}}{4}+\sqrt{1-\lambda^{2}}\left(1-\frac{9 \lambda^{2}}{4}\right)\right] \\
(0,0) & 0 & \frac{1}{\lambda}\left(1-\sqrt{1-\lambda^{2}}\right) \\
(-1,0) & -\frac{1}{4 \lambda}\left(1-\sqrt{1-\lambda^{2}}\right)^{2} & \frac{1}{4}\left(1+\sqrt{1-\lambda^{2}}\right) \\
(-1,-1) & 0 & \frac{1}{4 \lambda}\left[1+\frac{5 \lambda^{2}}{4}+\sqrt{1-\lambda^{2}}\left(1-\frac{\lambda^{2}}{4}\right)\right]
\end{array}
$$

Table 5: Level 0 and 1 matrix elements in the real case.

These results are also obtainable by means of overlap techniques as demonstrated in [25]. However, when comparing the structure of the vertex given in (87) to the corresponding expressions for real RNS fermions obtained in [25, 37] one should be aware of the fact that our vertex is not a state in the tensor product of four Hilbert spaces as is the case for the vertices in these references, but rather an operator in two Hilbert spaces. This feature makes it possible 
to view the whole vertex as a stringy extension of the tensor product of two Dirac matrices. Using coherent states one can, however, rewrite our vertex in the form of a state in the tensor product of four Hilbert spaces and thereby producing two extra terms in the exponent. These extra terms appear explicitly in e.g. [37].

\section{Conclusions}

In this paper we have sewn together two dual Ramond reggeon vertices to obtain an expression for the reggeon vertex with four external $R$ states. Since we are using dual vertices [33, 34, 7] the so obtained four-vertex is an operator in the tensor product of two Hilbert spaces. One unusual feature of the dual vertex used here is that it is normal ordered by means of two normal ordering fields (both of NS type) and is as a consequence universal for all choices of explicit t normal ordering and independent of whether we use real or complex fields. The actual sewing, i.e. performing of the infinite dimensional integral that results from insertion of coherent states, does depend on whether we use complex or real fields, and even on the specific form of the projective transformations used in defining the emission points. Results are presented for both complex and real fermions, and for four choices of projective transformations which makes the computation of the four-vertex tractable. We have not tried to carry out these calculations for more general transformations, but since the tricks of section 3 rely heavily on the choices made here (and which is basically the same as the ones used in all previous works on the subject), one might anticipate problems for any other choice of transformations.

The sewing produces twisted reggeon four-vertices which are expressed in terms of certain quantities that are built out of infinite dimensional matrices and vectors. These quantities depend on which fermionic oscillator modes they multiply in the exponent of the vertex and become increasingly complicated as one considers terms with higher and higher modes. So far, to our knowledge, only the quantity related to two zero modes has been derived. Here we show that these quantities can, in fact, be computed at any mode level and we present

\footnotetext{
${ }^{4}$ By explicit in this context we mean normal ordering prescriptions which do not involve any extra fields for their definition.
} 
explicit formulae for all those multiplying level zero and/or level one oscillators. These results are then compared to a closed form of the four-vertex the structure of which was first proposed in the case of three-vertices by LeClair [36]. The closed form of the four-vertex, discussed also in [37 but there it was verified to be correct only for the zero mode term, is easily seen to reproduce all our explicit sewing results and there can be no doubt that this situation will prevail for all modes. One of the nice features about this kind vertices is the way the branch cuts produced by the external twisted fields are implemented. This is done by expressing the vertex as the exponential of a double integral over two fermi fields (each one being a sum over all external (twisted or untwisted) fields present) connected by a propagator. This propagator is constructed by means of a correlation functions of spin fields and ordinary fermions distinct from the external ones. The spin fields in these correlators create the cuts necessary for the propagator to represent the cut surface correctly. The exact form of the vertex depends on the projective transformations chosen, at least in the complex case. However, a universal expression is obtained if the vertex is rewritten in terms of the projective transformations themselves as in eq. (89). It reads

$$
\begin{aligned}
\hat{W}_{R_{1} R_{2}}\left(V_{1}, V_{2}\right)= & \left(\frac{V_{2}^{-1}\left(z_{1}^{(1)}\right)}{V_{2}^{-1}\left(z_{2}^{(1)}\right)} \frac{V_{1}^{-1}\left(z_{1}^{(2)}\right)}{V_{1}^{-1}\left(z_{2}^{(2)}\right)}\right)^{\frac{d}{8}} \\
& \times: \exp \left(\oint_{C_{z}} d z \oint_{C_{w}} d w \hat{\psi}_{R}^{a V^{-1}}(z) \frac{\sqrt{\frac{V_{1}^{-1}(w) V_{2}^{-1}(w)}{V_{1}^{-1}(z) V_{2}^{-1}(z)}}}{z-w} \hat{\bar{\psi}}_{R}^{a V^{-1}}(w)\right):
\end{aligned}
$$

where we have utilized the observation that $\frac{V_{2}^{-1}\left(z_{1}^{(1)}\right)}{V_{2}^{-1}\left(z_{2}^{(1)}\right)}=\frac{V_{1}^{-1}\left(z_{1}^{(2)}\right)}{V_{1}^{-1}\left(z_{2}^{(2)}\right)}$ to rewrite the prefactor in a more symmetric fashion. This suggests of course a very direct extension to any number of twisted or untwisted external legs. First, the fermions in the exponent are then sums over all external (twisted or untwisted) fermions and the contours enclose all branch cuts and poles of the external fields. Also, the prefactor as well as the propagator in the exponent generalize in the obvious manner to an arbitrary number of branch cuts. In terms of spin field correlation functions the generalization is even more obvious as already observed in [37]: each new branch cut introduces a new pair of $\bar{S}\left(z_{1}\right) S\left(z_{2}\right)$ inside the correlator which then most likely gives the correct answer for both the propagator and prefactor. However, one should be aware of the 
fact that no proofs of any results along the lines of the ones in this paper exist for vertices with 6 or more external Ramond legs. In fact, in order to deal with more than four external legs one must understand how to repeat the manipulations of section 3 when more than one moduli parameter is involved. This problem is likely to be related to the problems that would appear were we to use transformations different from the ones used in this paper. 


\section{References}

[1] V. Alessandrini, D. Amati, M. Le Bellac and D. Olive, Phys. Reports 1 (1971) 269.

[2] C. Montonen, Nuovo Cimento 19A (1974) 69.

[3] P. Di Vecchia, R. Nakayama, J.L. Petersen, S. Sciuto and J.R. Sidenius, Nucl. Phys. B287 (1987) 621.

[4] J.L. Petersen, J.R. Sidenius and A.K. Tollstén, Nucl. Phys. B317 (1989) 109.

[5] P. Di Vecchia, M. Frau, K. Hornfeck, A. Lerda, F. Pezzella and S. Sciuto, Nucl. Phys. B322 (1989) 317.

[6] P. Di Vecchia, M. Frau, K. Hornfeck, A. Lerda, F. Pezzella and S. Sciuto, Nucl. Phys. B333 (1990) 635.

[7] N. Engberg, B.E.W. Nilsson and P. Sundell, Int. J. Mod. Phys. A7 (1992) 4559.

[8] E. Corrigan and D. Olive, Nuovo Cimento 11A (1972) 749.

[9] J. Schwarz, Nucl. Phys. B65 (1973) 131.

[10] E. Corrigan, Nucl. Phys. B69 (1974) 325.

[11] J.H. Schwarz and C.C. Wu, Phys. Lett. B47 (1973) 453.

[12] E. Corrigan, P. Goddard, D. Olive and R.A. Smith, Nucl. Phys. B67 (1973) 477.

[13] J.H. Schwarz and C.C. Wu, Nucl. Phys. B72 (1974) 397.

[14] J.H. Schwarz and C.C. Wu, Nucl. Phys. B73 (1974) 77.

[15] J. Schwarz, Nucl. Phys. B76 (1974) 93.

[16] E. Corrigan and D.B. Fairlie, Nucl. Phys. B91 (1975) 527.

[17] D. Bruce, E. Corrigan and D. Olive, Nucl. Phys. B95 (1975) 427. 
[18] P. Goddard and R. Horsley, Nucl. Phys. B111 (1976) 272.

[19] E. Corrigan and T.J. Hollowood, Nucl. Phys. B303 (1988) 135.

[20] E. Corrigan and T.J. Hollowood, Nucl. Phys. B304 (1988) 77.

[21] L. Dolan, P. Goddard and P. Montague, Nucl. Phys. B338 (1990) 529.

[22] A.A. Belavin, A.M. Polyakov and A.B. Zamolodchikov, Nucl. Phys. B241 (1984) 333.

[23] V.G. Knizhnik, Phys. Lett. B160 (1985) 403;

D. Friedan, E. Martinec and S. Shenker, Nucl. Phys. B271 (1986) 93.

[24] A. Neveu and P. West, Comm. Math. Phys. 114 (1988) 613.

[25] M.D. Freeman and P. West, Phys. Lett. B217 (1989) 259.

[26] E. D'Hoker and D.H. Phong, Rev. Mod. Phys. 60 (1988) 917.

[27] S. Mandelstam in Proceedings of the Workshop on Unified String Theories, Santa Barbara 1985, (World Scientific 1986), M.B. Green and D.J. Gross eds.

[28] L. Alvarez-Gaumé, C. Gomez, G. Moore and C. Vafa, Nucl. Phys. B303 (1988) 455;

L. Alvarez-Gaumé, P. Nelson, C. Gomez, G. Sierra and C. Vafa, Nucl. Phys. B311 (1988/89) 333.

[29] S. Sciuto, Lett. Nuovo Cimento 2 (1969) 411.

[30] A. Della Selva and S. Saito, Lett. Nuovo Cimento 4 (1970) 689.

[31] A. Neveu and P. West, Nucl. Phys. B278 (1986) 601; A. Neveu and P. West Phys. Lett. 179B (1986) 235.

[32] P. Di Vecchia, R. Nakayama, J.L. Petersen and S.Sciuto, Nucl. Phys. B282 (1987) 189. 
[33] B.E.W. Nilsson, A.K. Tollstén, A. Wätterstam and P. Hermansson, Phys. Lett. B236 (1990) 417.

[34] B.E.W. Nilsson and A.K. Tollstén, Phys. Lett. B240 (1990) 96.

[35] P. Hermansson, B.E.W. Nilsson, A.K. Tollstén and A. Wätterstam, Phys. Lett. B244 (1990) 209.

[36] A. LeClair, Nucl. Phys. B303 (1988) 189.

[37] P. Di Vecchia, K. Hornfeck, R. Madsen and K.O. Roland, Phys. Lett. B235 (1990) 63.

[38] B.E.W. Nilsson and P. Sundell, Göteborg preprint ITP 92-44 (1992). 